\title{
Operation of a scanning near-field optical microscope in reflection in combination with a scanning force microscope
}

Niko F. van Hulst, Marco H. P. Moers, Oscar Noordman, T. Faulkner, F. B. Segerink, et al.

Niko F. van Hulst, Marco H. P. Moers, Oscar Noordman, T. Faulkner, F. B. Segerink, Kees O. van der Werf, Bart G. de Grooth, Bouwe Bolger, "Operation of a scanning near-field optical microscope in reflection in combination with a scanning force microscope," Proc. SPIE 1639, Scanning Probe Microscopies, (1 May 1992); doi: 10.1117/12.58190 


\title{
Operation of a scanning near field optical microscope in reflection in combination with a scanning force microscope
}

\author{
N.F. van Hulst, M.H.P. Moers, O.F.J. Noordman, T. Faulkner, F.B. Segerink, \\ K.O. van der Werf, B.G. de Grooth \& B. Bölger \\ Department of Applied Physics, University of Twente, \\ P.O.Box 217 7500AE Enschede, the Netherlands
}

\begin{abstract}
Images obtained with a Scanning Near field Optical Microscope (SNOM) operating in reflection are presented. We have obtained the first results with a $\mathrm{SiN}$ tip as optical probe. The instrument is simultaneously operated as a Scanning Force Microscope (SFM). Moreover the instrument incorporates an inverted light microscope (LM) for preselection of a scan area. The SiN probe is operated in the contact regime causing a highly improved lateral resolution in the optical image compared to an alternative set-up using a fibre probe, which is also presented. The combined microscope is operated either in open loop or as a force regulated SNOM. Near field optical images can be directly compared with the topography displayed in the simultaneously recorded SFM image.
\end{abstract}

\section{INTRODUCTION}

A major difference of Scanning Near field Optical Microscopy (SNOM), compared to STM and SFM, is the fact that the interaction is optical and consequently all techniques of conventional optical microscopy, e.g. detection of luminescence and polarization effects, can be adapted to SNOM provided a sufficient signal. However, the potential of SNOM depends strongly on the fabrication of efficient and reliable probes. This problem has stimulated the development of several types of near field probe tips over the last years.

The basic probe, developed by Pohl et al. ${ }^{1,2}$, is a sharpened dielectric tip coated with metal, leaving an aperture at the apex, $20 \mathrm{~nm}$ diameter being a practical lower limit. Betzig et al. ${ }^{3}$ have improved these probes by pulling adiabatically tapered single mode fibres which enable to illuminate the aperture with a higher efficiency. With these probes $12 \mathrm{~nm}$ lateral resolution and polarization contrast has been demonstrated. Recently Tortonese et al. ${ }^{4}$ have succeeded in fabricating single crystal silicon apertures down to $250 \mathrm{~nm}$. The application of aperture probes is limited to samples with gentle surface roughness as the outer diameter at the apex is generally larger than $250 \mathrm{~nm}$.

An interesting alternative probe type is formed by micro-pipettes, pulled to an inner diameter of about $50 \mathrm{~nm}$ and subsequently coated with metal. Betzig et al. ${ }^{5}$ have obtained a lateral resolution of about $50 \mathrm{~nm}$ using these tips as detection probe. Lewis et al. ${ }^{6}$ have filled these metal coated pipettes with fluorescent dye, thus generating a miniature fluorescence light source with relatively high brightness. Again these probes are restricted to flat samples.

An alternative approach based on localized frustrated total internal reflection has been described by Reddick ${ }^{7}$, Courjon 8 and our group ${ }^{9,10}$. In this case an evanescent field is generated at the sample surface by total internal reflection. The evanescent field is frustrated by a sharp dielectric probe and converted into a propagating wave. A lateral resolution down to $30 \mathrm{~nm}$ has been observed ${ }^{9}$. This alternative probe circumvents the problem of aperture fabrication, however it is restricted to transparent samples and rather sensitive for radiatively scattered waves ${ }^{10}$.

Recently Fischer 11 has presented the concept of an optical coaxial line, i.e. an optical fibre with a metal core. This type of probe seems promising in view of its expected efficiency. 
In the first part of this paper images obtained with a SNOM operated in reflection are presented, where the optical probe is a sharpened fibre. The microscope is applied to compact disc test structures, scanned at constant height. Distinct differences between the optical image and the surface topography have been observed, e.g. the reflected signal is enhanced when scanning over pits. The lateral resolution is about $100 \mathrm{~nm}$, mainly limited by tip sharpness and minimum distance between tip and surface.

All optical probes mentioned above have their specific limitations: generally a single tip touch is fatal, the efficiency is low, tips are hard to reproduce and samples should be flat. Yet for high resolution near field optical imaging close contact scanning at a distance below $20 \mathrm{~nm}$ is essential. On arbitrary surfaces this can only be accomplished by keeping the force between probe and sample low enough $(<1 \mu \mathrm{N})$ in order not to destroy the optical tip. We have realized an alternative SNOM where we take advantage of the unique features of the micro fabricated SiN probes commonly used in SFM. Hereto it should be noted that $\mathrm{SiN}$, besides its ideal properties for micro fabrication and its mechanical strength, is also an ideal optical material $(n=2.0)$ with transparency down to $290 \mathrm{~nm}$. Thus the $\mathrm{SiN}$ probe is a high index optical structure with $20-50 \mathrm{~nm}$ apex, very similar to the probe types mentioned previously, with the advantage that it is integrated with a cantilever and can be scanned in close contact over a surface without damage.

In the second part of this paper we present preliminary results of near field optical imaging in a modified SFM using a micro-fabricated $\mathrm{SiN}$ probe with the $\mathrm{SiN}$ tip itself as an optical probe. The inner side of the SiN tip is illuminated and the reflected light is detected, thus constituting a reflection SNOM. Simultaneously the cantilever deflection is detected for force imaging. The combined SNOM-SFM microscope has been applied to the same compact disc test structures.

\section{THE EXPERIMENTAL SET-UP}

\subsection{Reflection SNOM with fibre probe}

In this set-up the probe is formed by a fibre, sharpened by chemical etching in HF 9 . A light beam is coupled into the cleaved end of the fibre and reflected at the sharpened end where the dimensions are below the cut-off size. An evanescent field is generated at the end of the fibre itself. Simultaneously a small fraction of the light is emitted as a radiative wave. A dielectric surface brought into close proximity frustrates the evanescent wave and part of the radiative wave is reflected, both affecting the effective intensity of the reflected light. The microscope is sketched in Fig. 1. The light source is a laser diode $(5 \mathrm{~mW}, 780 \mathrm{~nm})$ with short coherence length in order to avoid the contribution of interference between the fibre ends and collimating optics. The light reflected from the tip $(\sim 1 \mu \mathrm{W})$ is directed towards a photo diode via a beam splitter.

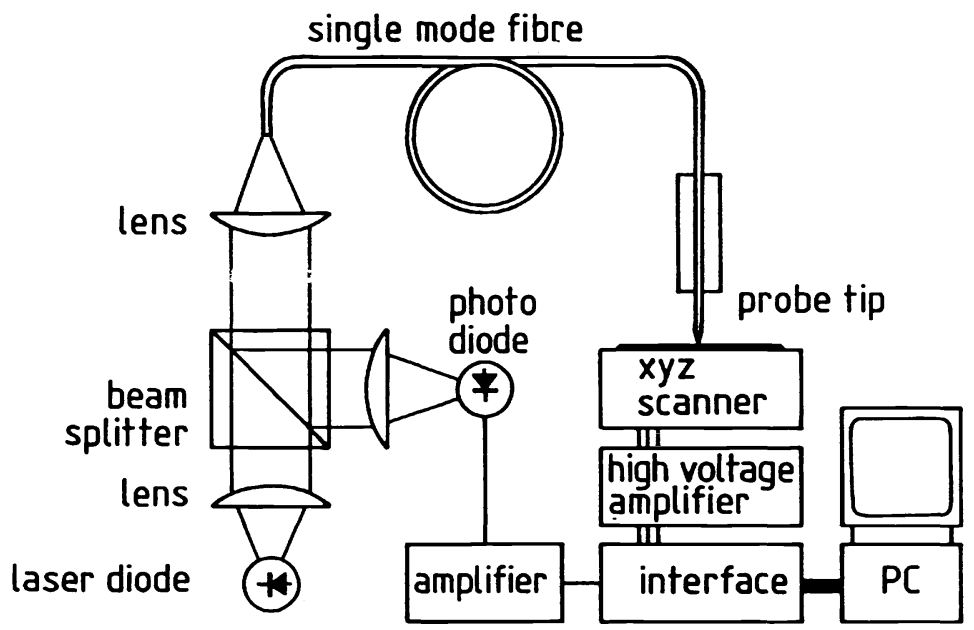

Figure 1: Experimental set-up of the reflection microscope with fibre probe. 
The sample is mounted on a commercial manipulator stage (Photon Control) with both mechanical and piezo-electric adjustments in $\mathrm{x}, \mathrm{y}$ and $\mathrm{z}$ directions. The presence of a sample changes the reflected signal less than $1 \%$ for transparent surfaces to up to $10 \%$ for highly reflecting surfaces. The reflection signal is passed through a high-pass filter before further amplification in order to remove the DC offset. The cut-off frequency $(0.1 \mathrm{~Hz})$ is low enough compared to the line frequency $(2-10 \mathrm{~Hz})$ in order not to differentiate the signal. An image is obtained by scanning the sample at constant distance to the tip, without feedback. A similar "external reflection" microscope using a HeNe laser and $3 \mathrm{~dB}$ fibre couplers has been described by Courjon et al. ${ }^{8,12}$.

\subsection{Combined SNOM-SFM-LM with integrated SiN probe}

The set-up is sketched in Fig. 2. The microscope is based on the design of a scanning force microscope with integrated optical microscope as developed by Putman et al. ${ }^{13}$. The heart of the instrument is a commercial micro fabricated SiN cantilever with integrated pyramidal tip ${ }^{14}$, where the cantilever is placed at $15^{\circ}$ angle to the sample surface. The position of the cantilever is fixed during operation, while the sample is scanned using a piezo-electric tube.

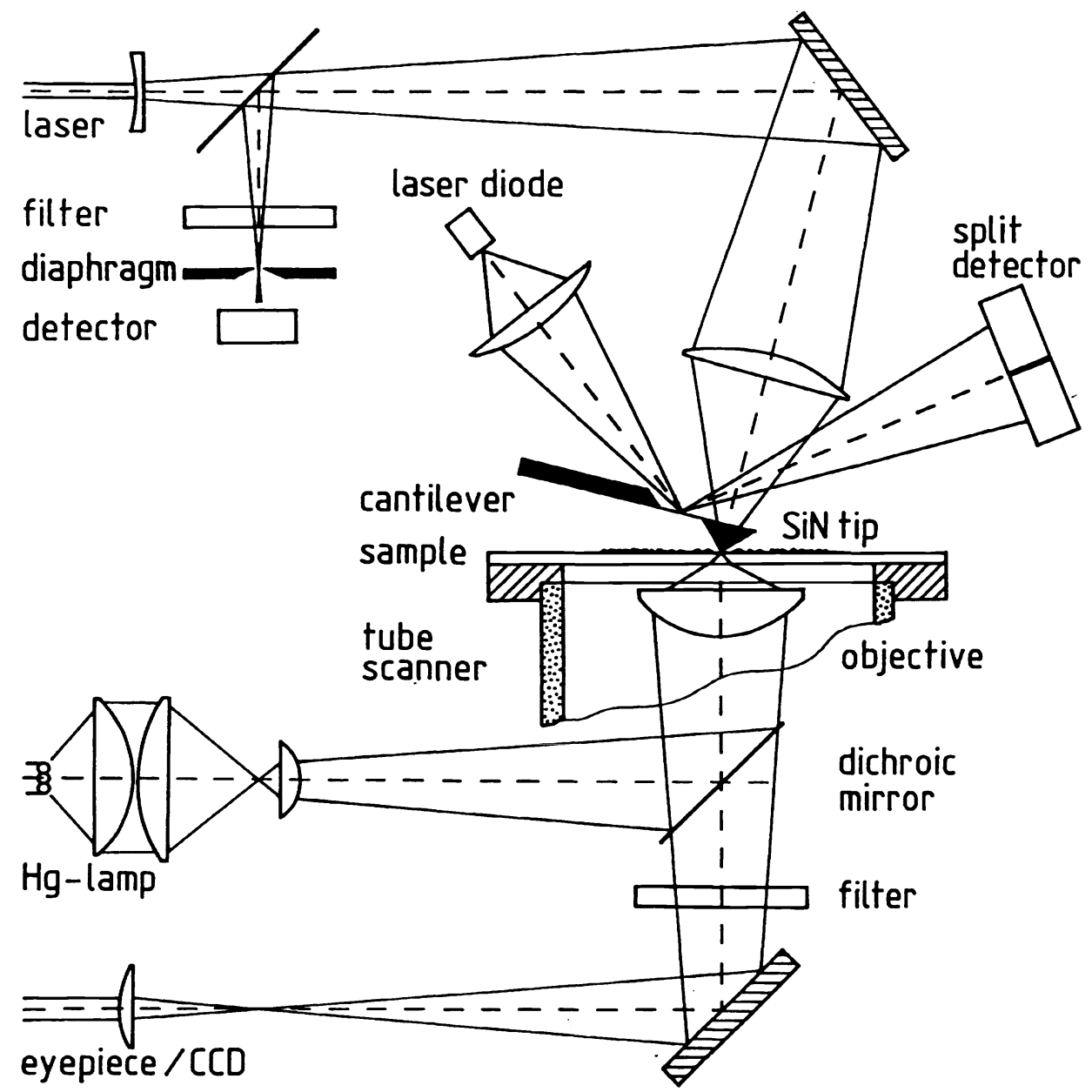

Figure 2: Experimental set-up of the combined SNOM (top) - SFM (middle) based on a SiN cantilever, combined with an inverted epi-illumination light microscope (LM, bottom). For clarity the size of the SiN cantilever has been exaggerated. 
In Fig. 2 three different optical paths should be distinguished :

(i) SNOM-system : Above the cantilever is a lens which focuses a HeNe laser beam at normal incidence into the $\mathrm{SiN}$ tip. The laser spot at the cantilever has $2-3 \mu \mathrm{m}$ diameter, which fits within the $3 \times 3 \mu \mathrm{m}$ bottom facet of the $\mathrm{SiN}$ pyramidal tip and is thus illuminating the inner side of the $\mathrm{SiN}$ apex. A magnified image of the tip in reflection is formed outside the microscope head. A diaphragm in the imaging plane is adjusted such that only the beam reflected at the centre of the tip is passed onto a photo diode. Part of the detected light intensity $(\sim 1 \mu \mathrm{W})$ comes from direct reflection at the $\mathrm{Au}$ coating on the back of the cantilever. At the apex of the illuminated tip we expect an evanescent field, caused by total internal reflection, while part of the light is transmitted as a radiative wave.

(ii) SFM-system : Close to the cantilever, within the microscope head, is an optical beam deflection system (Meyer et al. ${ }^{15}$ ) for detection of the interaction force between tip and sample. The laser diode $(3 \mathrm{~mW}, 780 \mathrm{~nm})$ is focused onto the cantilever at $30 \circ$ incidence angle and the reflected beam is detected on a split detector. In fact the detector is a 4-segment photo diode which enables separate detection of cantilever deflection and cantilever torsion.

(iii) LM-system: Under the sample is an inverted optical microscope with epi-illumination. The objective, a commercial CD-player lens with $0.45 \mathrm{NA}$, is mounted within the tube and can be manipulated independently in $\mathrm{x}, \mathrm{y}$ and $\mathrm{z}$ directions. The illumination source is a $100 \mathrm{~W} \mathrm{Hg}$ arc lamp. The LM facilitates simultaneous viewing of the sample, the position of the tip over surface, the position of the HeNe beam in the SiN pyramid and the position of the laser diode spot on the cantilever. Thus the LM enables preselection of a required scan area and alignment of the HeNe beam focus within the $\mathrm{SiN}$ tip.

The instrument is operated in air, in the contact mode, either in open loop or at constant force with a feedback on the optical beam deflection signal. Operation with released tip is also possible, however, the minimum distance out of contact is $\geq 100 \mathrm{~nm}$ due to attractive surface tension forces caused by the water film on the surface (Weisenhorn et al. ${ }^{16}$ ).

\section{SAMPLE}

The performance of the microscopes has been verified on compact disc test structures, obtained from Philips Research Labs. the Netherlands. Figures 3 a \& b show images of the CD-structure as observed with the LM. The polycarbonate structure, fabricated by replication from a stamper, consists of indented tracks varying from dotted patterns to long trenches. The track spacing is $1.25 \mu \mathrm{m}$. The trench width is $175 \pm 25 \mathrm{~nm}$.

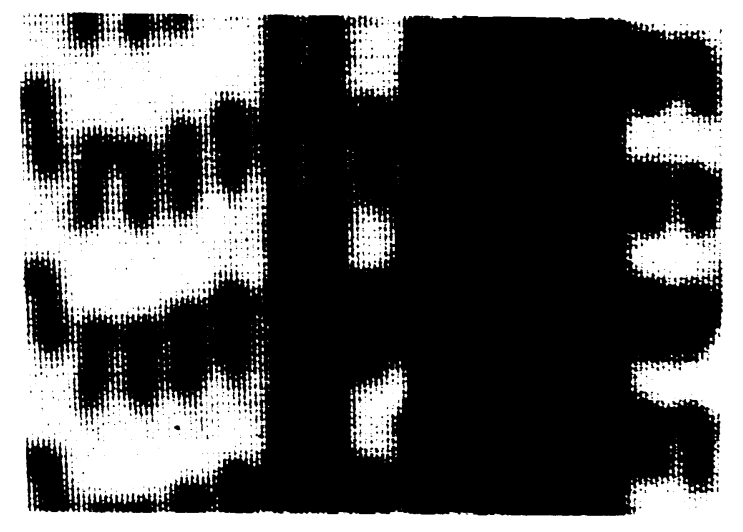

(a)

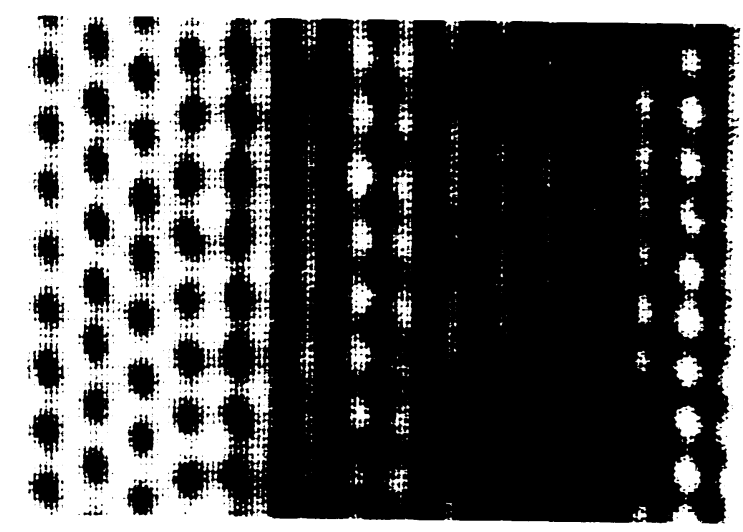

(b)

Figure 3: $18 \times 13 \mu \mathrm{m}$ area of the compact disc test structure observed with the inverted optical microscope, displaying trenches, pits of 2.0 m length (a) and circular pits (b). 


\section{RESULTS}

\subsection{Images with the fibre probe system}

When decreasing the distance between the fibre tip and the sample surface a characteristic Fabry Perot fringe pattern is observed, caused by interference between tip and sample. The fringe amplitude is increasing rapidly towards the surface, down to a distance below $\lambda / 2$, where an abrupt deviation from the fringe pattern is observed, corresponding to the near field regime. The deviation is rather variable depending on the sample surface and the fibre tip used.
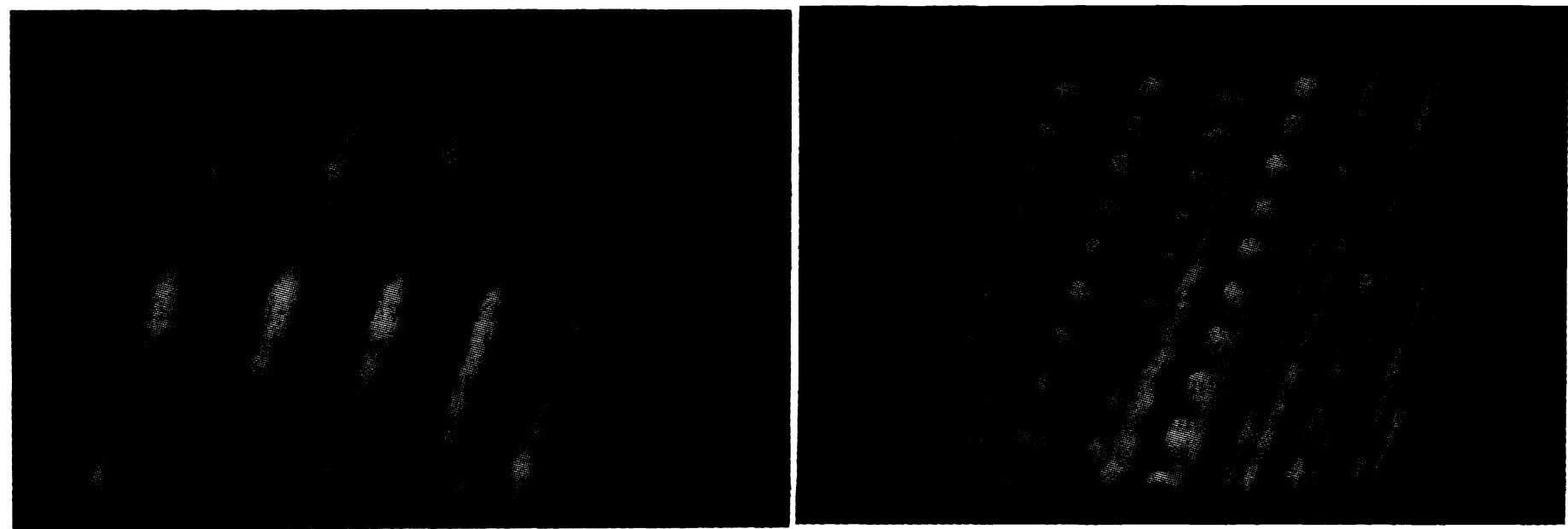

Figure 4a: $7 \times 7$ um image of the reflected signal over a structure with $\mathrm{Ag}$ coated pits of $2.0 \mu \mathrm{m}$ length and $1.25 \mu \mathrm{m}$ period.

Figure 4b: $9 \times 9 \mu m$ scan over an area with $\mathrm{Ag}$ coated circular pits $(200 \mathrm{~nm}$ diameter $)$ and trenches, both with 1.25 um period.

Fig. 4a shows a $7 \times 7 \mu \mathrm{m}$ 3D image of the reflection signal over a part of the area displayed in Fig. 3a, scanned at a constant height of about $100 \mathrm{~nm}$. The CD pits have been coated with $\mathrm{Ag}$ for higher reflectivity. The actual pits are $2.0 \mu \mathrm{m}$ long and $175 \mathrm{~nm}$ wide. The pits stand out on their surrounding area as they appear to enhance the reflected signal. This has been observed also at larger distances up to $3 \mu \mathrm{m}$, be it with less intensity. It appears that the pits enhance the total level of reflected signal, independent of the distance dependent fringes on top of the it. Probably the pits act as focusing objects. The signal level directly beside the pits is slightly below the average signal level of the surrounding area. This feature disappears when increasing the distance. The observed width of the pits is about $500 \mathrm{~nm}$ and increases with the working distance. The lateral resolution is about $100 \mathrm{~nm}$, mainly determined by the sharpness of the tip and the non-zero distance and the effective acceptance angle of the tip for reflected light. In the upper right corner of Fig. $4 \mathrm{a}$ are interference fringes with $\lambda / 2$ period.

Fig. 4b shows a $9 \times 9 \mu \mathrm{m}$ reflection scan over an area as in Fig. 3b, displaying both circular pits and long trenches. Again the indented pits enhance the reflected signal. They stand out as bumps with $-500 \mathrm{~nm}$ diameter, while the actual size is $\sim 200 \mathrm{~nm}$. The trenches appear as a slight decrease in the average reflectivity, whereas the intermediate region appears more pronounce with stronger signal decrease. The appearance of the trenches is probably dominated by mutual interference of the reflected light from adjacent trenches.

We have found that the images depend on the shape of the etched fibre tip. Although the general features reproduce, distinct asymmetric structures can be observed from tip to tip.

Line scans over a grating by Courjon et al. ${ }^{8,12}$ display similar fringe-like patterns superimposed over the actual structure and enhanced reflection signal in the grating grooves. Girard et al. ${ }^{17}$ have evaluated a theoretical model indicating that spurious waves emitted from the tip could also account for the oscillatory patterns observed in reflection imaging. 
Force

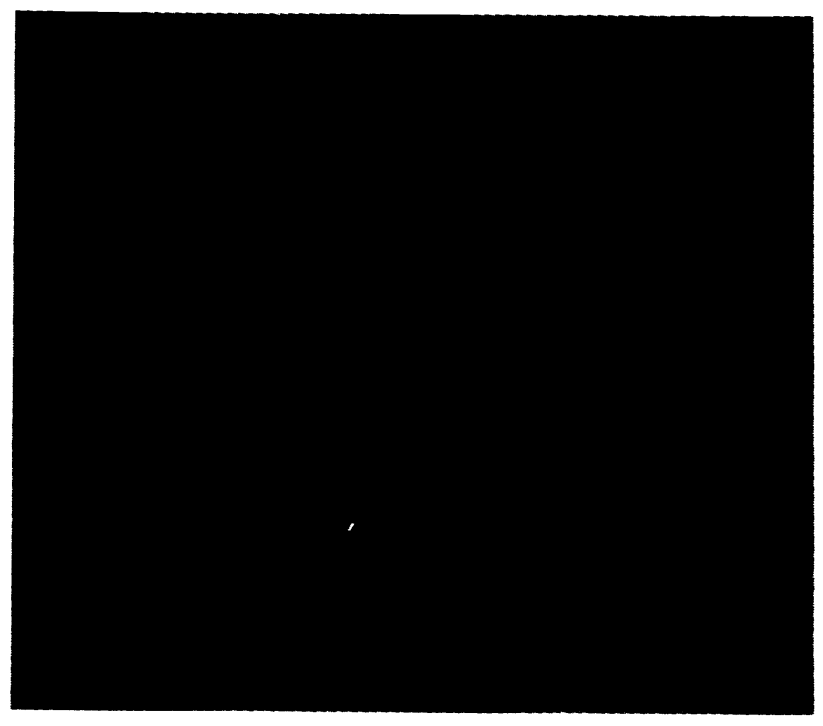

(a)

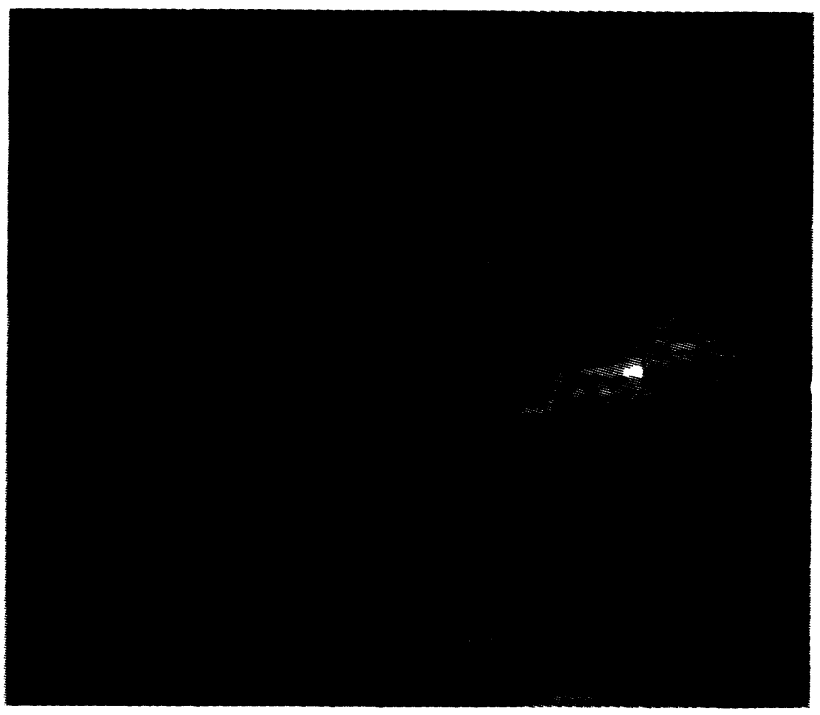

(c)

Figure 5: $6.5 \times 6.5 \mu \mathrm{m}$ scans over a CD structure with tracks and pits:

(a) SFM image in contact mode. The microscope is scanned in open loop and the force signal is displayed.

(b) optical signal recorded simultaneously with $(a)$.

(c) SFM image in contact mode. The microscope is scanned in closed loop and the feedback signal is displayed.

(d) optical signal recorded simultaneously with (c).

(e) optical signal recorded with the tip released from the sample surface. The corresponding SFM image is not displayed as no force effect can be observed. (d)

Optical

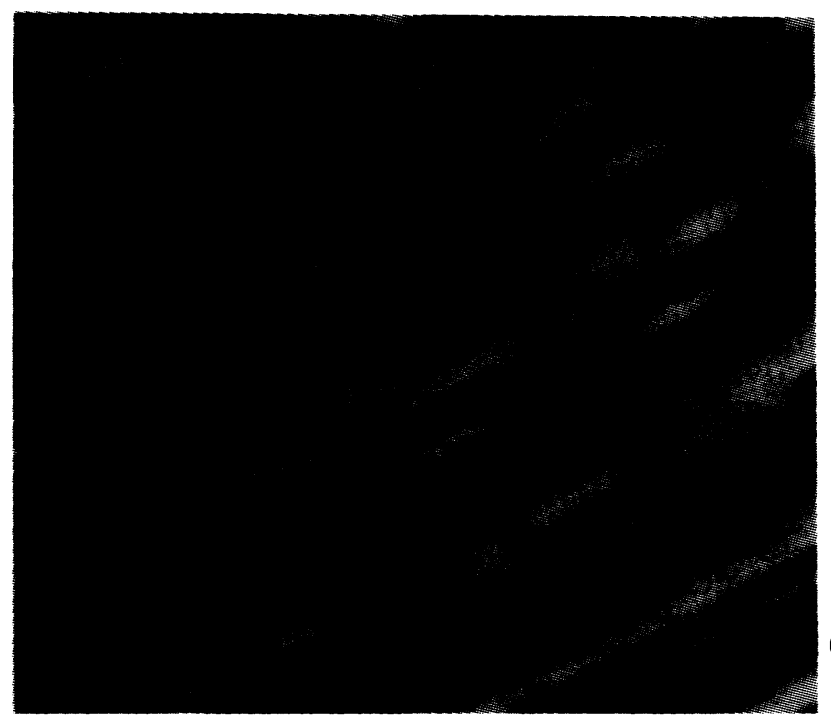

(b)
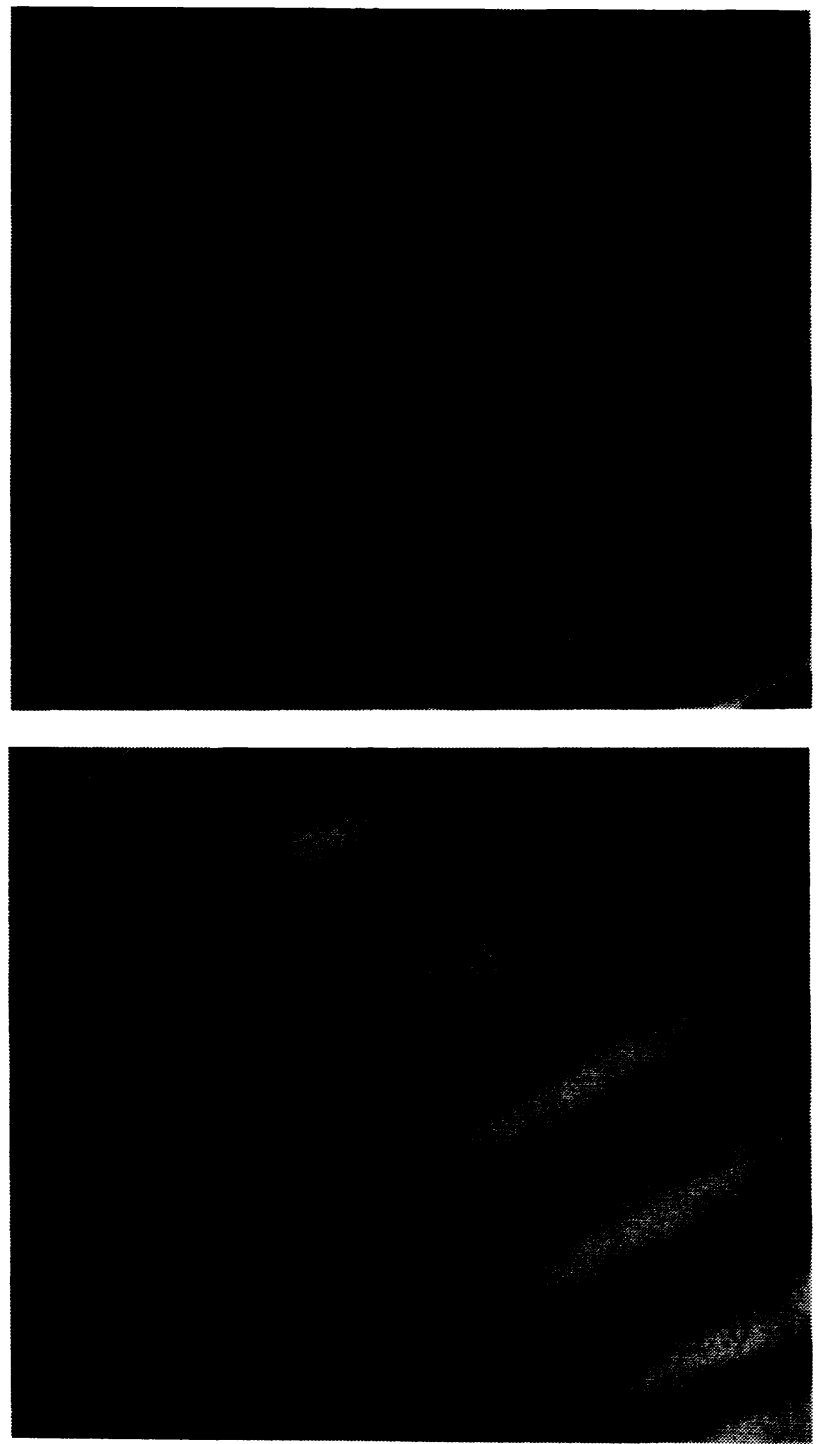

(e) 


\subsection{Images with the SiN probe system}

Fig. 5 shows images as obtained with the combined SNOM-SFM system. An area on the polycarbonate structure was scanned similar to the area displayed in Fig. 3a., with trenches and pits of $1.0 \mu \mathrm{m}$ length.

Figures 5a and 5b display the simultaneously recorded SFM and optical image, respectively, as obtained in close contact without feedback on the force signal. Thus the cantilever follows freely the underlying structure. The force image corresponds to the topographic structure, where the finest detail of $-30 \mathrm{~nm}$ is determined by the tip sharpness. The white lines indicate a short release of the tip during a line scan. The optical image (Fig. 5b) corresponds to changes in the effective reflectivity. The signal is enhanced at the rim of the trenches and pits. The intermediate regions show smooth signal variation, correlated to the tracks. The fine corrugation, present in the force image, has disappeared in the optical image. The bending of the cantilever could influence the optical reflection signal, resulting in an SFM-like contribution. If present, this contribution can be largely reduced by operating in feedback. To verify this we have performed a similar scan, in close contact, this time with a feedback on the force signal. Thus the sample height is corrected according to the detected force, while the cantilever is not bending within the bandwidth of the feedback system. The simultaneously recorded SFM and optical image are displayed in Figures 5c and 5d, respectively. The SFM image in Fig. 5c, displaying the feedback signal, corresponds to the topographic structure as also observed in Fig. 5a. The optical image in Fig. 5d is very similar to Fig. 5b, except for some higher spatial frequency details, indicating that the effect of bending of the cantilever on the optical image is limited. Finally, in order to verify the effect of any other residual force, we have performed a scan with the tip released. Fig. 5e displays the image, which now consists of purely optical effects. The distance between tip and sample is at least $100 \mathrm{~nm}$, in order not to be attracted by the surface tension force. The cantilever deflection was below the detection limit and consequently a corresponding SFM image is not displayed. Fig. 5e still shows the underlying track structure, however any fine structure is lost. Moreover contrast appears to reverse from left to right.

It should be noted that the optical images in figures $5 \mathrm{~b}, \mathrm{~d}$ and e originate from the $\mathrm{SiN}$ tip as they disappear when the HeNe beam is focused beside the pyramid. The optical signal is of comparable amplitude in open loop, closed loop and with released tip. Positioning of the focus in the pyramid is critical and affects the observed images.

Interpretation of figures $5 \mathrm{~b}, \mathrm{~d}$ and $\mathrm{e}$ is not straightforward. Based on the experiments we suppose that the detected optical signal consists of the reflected light at the apex, an interference contribution and some residual effects of cantilever bending. Obviously further research should be performed to clarify aspects.

\section{CONCLUSIONS}

Both types of microscopes presented are capable of optical imaging with structural details surpassing the optical diffraction limit.

The fibre probe system is easy to operate, however the fibre tip is a weak point. Images are mainly determined by geometrical optical effects and interference of radiative waves.

We have obtained the first optical images using the integrated $\mathrm{SiN}$ probe. The probe is highly promising for high resolution optical imaging as it can be scanned in close contact without damage. Moreover it can simultaneously be operated as an SFM which enables force regulation and direct comparison with the force images. The optical images display structural features down to $50-100 \mathrm{~nm}$. Further experiments are necessary to interpret the presented images.

\section{ACKNOWLEDGEMENTS}

This research was mainly supported by the Dutch Foundation for Fundamental Research on Matter FOM. We thank K. Smit for the mechanical construction of the integrated microscope. 


\section{REFERENCES}

1. U. Dürig, D.W. Pohl \& F. Rohner, Near-field optical-scanning microscopy. J. Appl. Phys. $\underline{59}$, 3318-3327 (1986).

2. U.Ch. Fischer, U.T. Dürig \& D.W. Pohl, Near-field optical scanning microscopy in reflection. Appl. Phys. Lett. 52, 249-251 (1988).

3. E. Betzig, J.K. Trautman, T.D. Harris, J.S. Weiner \& R.L. Kostelak, Breaking the Diffraction Barrier: Optical Microscopy on a Nanometric Scale. Science 251, 1468-1470 (1991).

4. M. Tortonese, H. Yamada \& C.F. Quate, Microfabricated probe for a near field scanning optical microscope using a force detection scheme. Poster presentation at STM'91 Interlaken (1991).

5. E. Betzig, M. Isaacson \& A. Lewis, Collection mode near-field optical microscopy. Appl. Phys. Lett. 51, 2088-2090 (1987).

6. A. Lewis \& K. Lieberman, Near field optical imaging with a non-evanescently excited highbrightness light source of sub-wavelength dimensions. Nature 354, 214-216 (1991).

7. R.C. Reddick, R.J. Warmack, D.W. Chilcott, S.L. Sharp \& T.L. Ferrell, Photon Scanning Tunneling Microscopy. Rev. Sci. Instrum. 61, 3669-3677 (1990).

8. D. Courjon, J.-M. Vigoureux, M. Spajer, K. Sarayeddine \& S. Leblanc, External and internal reflection near field microscopy: experiments and results, Appl. Opt. 29, 3734-3740 (1990).

9. N.F. van Hulst, F.B. Segerink \& B. Bölger, High resolution imaging of dielectric surfaces with an Evanescent Field Optical Microscope. Opt.Comm. in press.

10. N.F. van Hulst, F.B. Segerink, F. Achten \& B. Bölger, Evanescent Field Optical Microscopy: effects of polarization, tip shape and radiative waves. Proceedings STM'91, Accepted for Ultramicr. in press.

11. U.Ch. Fischer, The wire probe for Scanning Near field Optical Microscopy (SNOM), presented at STM'91, Interlaken (1991).

12. D. Courjon, M. Spajer, Reflection Near-field Optical Microscopy. J. Phys. III 1, 1-12 (1991).

13. C.A.J. Putman, K.O. van der Werf, B.G. de Grooth, N.F. van Hulst, F.B. Segerink \& J. Greve, Atomic force microscope with integrated optical microscope for biological applications. Rev. Sci. Instr. in press.

14. Park Scientific Instruments, Sunnyvale, California.

15. G. Meyer \& N.M.Amer, Novel optical approach to atomic force microscopy. Appl. Phys. Lett. $\underline{53}$, 1045-1047 (1988).

16. A.L. Weisenhorn, P.K. Hansma, T.R. Albrecht \& C.F. Quate, Forces in atomic force microscopy in air and water. Appl. Phys. Lett 54, 2651-2653 (1989).

17. C. Girard \& M. Spajer, Model for reflection near field microscopy, Appl. Opt. 29, 3726-3733 (1990). 